\title{
WestVirginiaUniversity
}

THE RESEARCH REPOSITORY @ WVU

Graduate Theses, Dissertations, and Problem Reports

2021

\section{The Impact of Driver Reaction in Cooperative Vehicle Safety Systems}

\author{
Elwarfalli Ibrahim \\ ieelwarfalli@mix.wvu.edu
}

Follow this and additional works at: https://researchrepository.wvu.edu/etd

Part of the Electrical and Computer Engineering Commons

\section{Recommended Citation}

Ibrahim, Elwarfalli, "The Impact of Driver Reaction in Cooperative Vehicle Safety Systems" (2021). Graduate Theses, Dissertations, and Problem Reports. 10208.

https://researchrepository.wvu.edu/etd/10208

This Problem/Project Report is protected by copyright and/or related rights. It has been brought to you by the The Research Repository @WVU with permission from the rights-holder(s). You are free to use this Problem/Project Report in any way that is permitted by the copyright and related rights legislation that applies to your use. For other uses you must obtain permission from the rights-holder(s) directly, unless additional rights are indicated by a Creative Commons license in the record and/ or on the work itself. This Problem/Project Report has been accepted for inclusion in WVU Graduate Theses, Dissertations, and Problem Reports collection by an authorized administrator of The Research Repository @ WVU. For more information, please contact researchrepository@mail.wvu.edu. 


\title{
The Impact of Driver Reaction in Cooperative Vehicle Safety Systems
}

\author{
Ibrahim E. Elwarfalli
}

\author{
Problem Report submitted to the \\ College of Engineering and Mineral Resources \\ at West Virginia University \\ in partial fulfillment of the requirements \\ for the degree of \\ Master of Science \\ in \\ Computer Science \\ Hany Ammar, Ph.D., Chair \\ Mohamed Hefeida, Ph.D. \\ Dale G. Dzielski, M.B.A., PMP, CMA \\ Lane Department of Computer Science and Electrical Engineering \\ Morgantown, West Virginia \\ Morgantown, West Virginia \\ 2021
}

Keywords: Driver Reaction, Cooperative Vehicle Safety Systems

Copyright 2018 Ibrahim Elwarfalli 


\section{Abstract \\ The Impact of Driver Reaction in Cooperative Vehicle Safety Systems}

\section{Ibrahim Elwarfalli}

Cooperative Vehicular Safety (CVS) has recently been widely studied in the field of automated vehicular systems. CVS systems help decrease the rates of accidents. However, implementing and testing CVS applications in real world is very costly and risky. Hence, most of the related research studies on CVS applications have relied mainly on simulations. In simulated CVS systems, it is important to consider all critical aspects of used models, and how these models affect one another.

The movement model is a key component in the simulation study of CVS systems, which controls the mobility of vehicles (nodes) and responses to the continually changing acquired information. However, existing mobility models are not created to take action(s) in response to hazardous situations (identified by situational awareness component). Integrating the reaction(s) to a hazardous alert is a missing element in current CVS system simulations. Hence to rectify this deficiency, this work is to incorporate a Driver's Reaction Model (DReaM) that react and respond to hazard alerts, and studies the effect of main components of CVS system including the added model. We examined a simulation modeling framework that describes cooperative vehicle safety system as one unified model. The studied framework is powered by cooperation and communication between vehicles. Investigated elements are communication model, movement model, warning generation, and driver response to warning indicating an emergency of near to crash situation. 


\section{Acknowledgements}

I take this opportunity to thank my advisor and committee chair Dr. Hany Ammar for his valuable guidance and relentless support. He inspired me greatly in working through my graduate study. I am grateful to Dr. Yaser Fllah for providing me an opportunity to work in his research lab. They were always inspiring and the knowledge they shared helped me a lot throughout my studies. I thank Dr. Mohamed Hefeida and Mr. Dale Dzielski for being a part of my committee.

I am thankful to Dr. Randall Jackson for providing me an opportunity to work as a Graduate Service Assistant at RRI, WVU. I also thank the Lane Department of Computer Science and Electrical Engineering for providing me resources and financial support through teaching and research assignments.

Also, I would like to thank my family and friends for their love and support. They were

always cordial and encouraged me in my work. Last but not the least, I give thanks to the Almighty Allah for answering my prayers and giving me strength at difficult times. 


\section{Table of Contents}

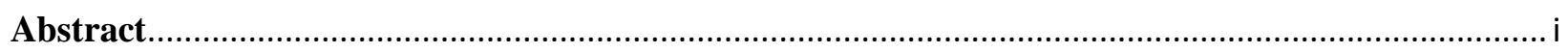

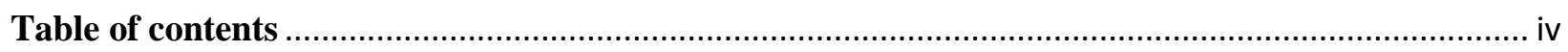

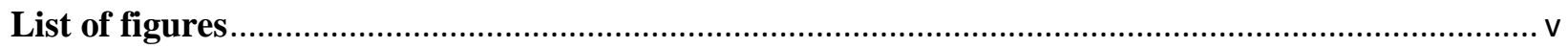

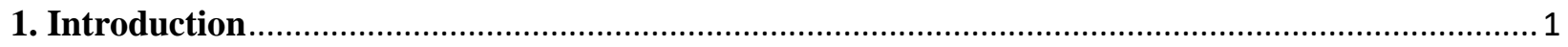

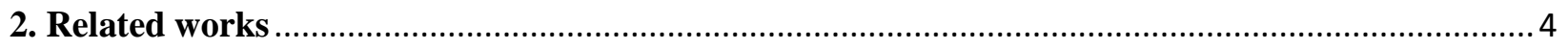

2.1 Related work on mobility model and mobility generator...................................................... 6

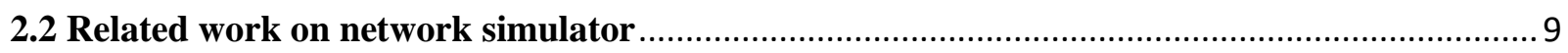

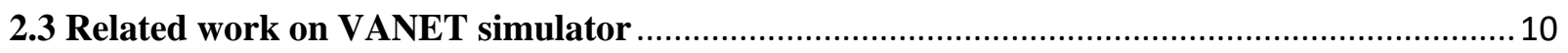

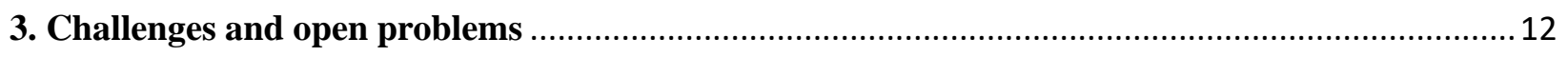

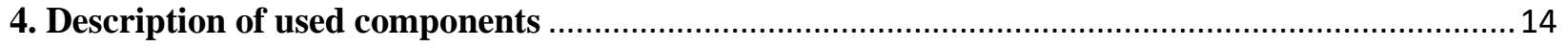

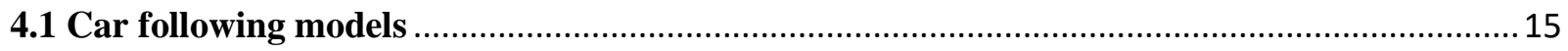

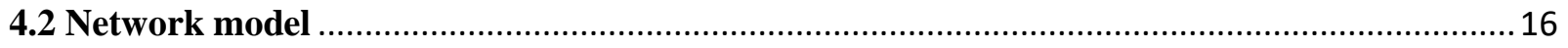

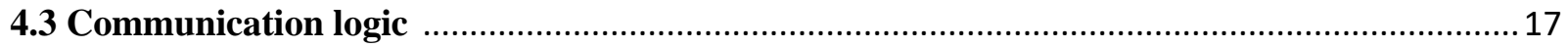

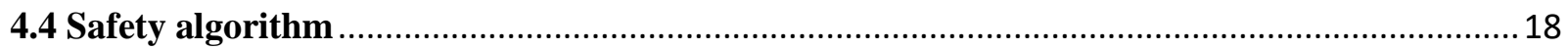

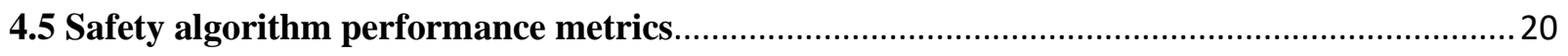

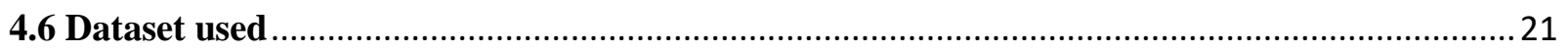

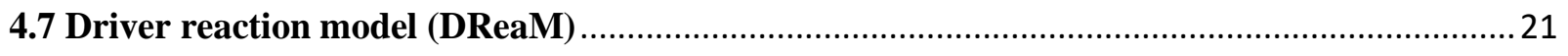

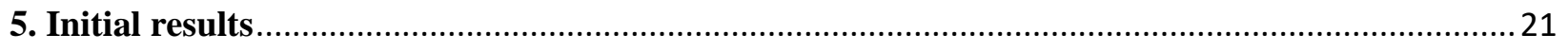

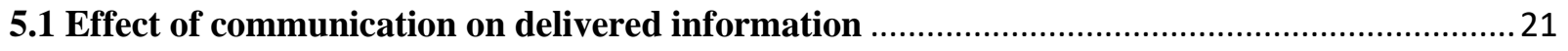

5.2 Effect of communication on position estimation of leading vehicle .......................................22

5.3 Effect of communication on performance of hazard detection algorithm .............................2 23

5.3 Effect of both networking and dream on vehicles movement and positioning of vehicles .......25

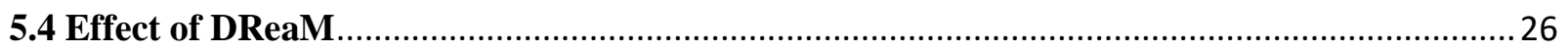

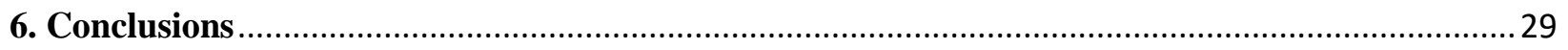

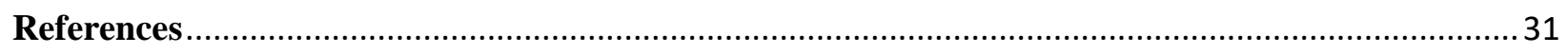




\section{List of Figures}

Figure 1. To enable CVS system, each vehicle broadcast its own state info via DSRC ............................ 2

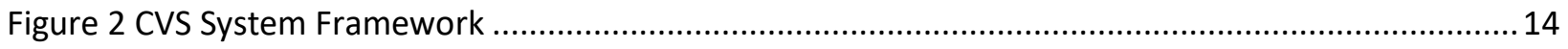

Figure 3. Communication logic: (top) Periodic Beaconing, (bottom) Network- Aware Error-Dependent. 18

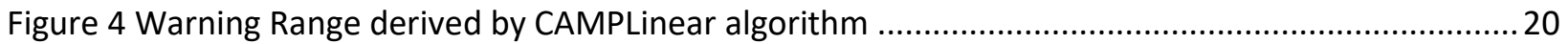

Figure 5 the effect on received acceleration and velocity data..............................................................22

Figure 6 Effect of networking on Estimation of Leading vehicle's Position ............................................23

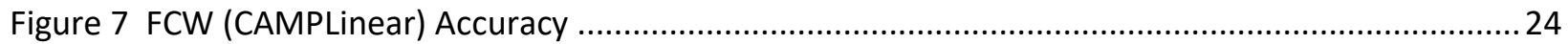

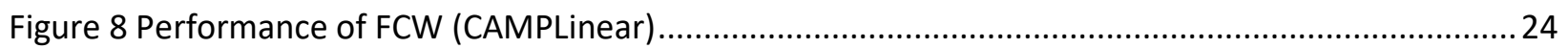

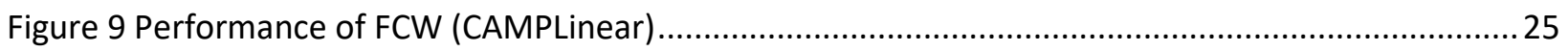

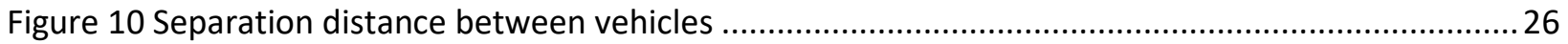

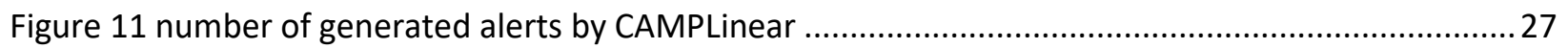

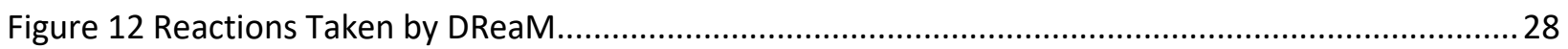

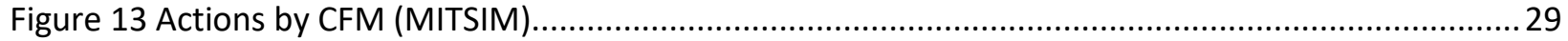




\section{Introduction}

Automotive safety systems have been widely studied by the research community. One of the main objectives of the research is to develop mechanisms for crash avoidance. Different collision avoidance systems have been proposed and developed. However, more work is needed to develop fully automated systems that are effective in all situations.

In order to evaluate decisions and choose between issuing a warning to driver or directly taking action to prevent a crash, collision avoidance systems depend on real-time mapping of surroundings of a vehicle (situational awareness). Situational awareness could be fed by data that is received from local sensors, or received from a wireless network of other vehicles, or received from both [2].

In cooperative safety system, it is essential for vehicles to always monitor any threats that could be caused by their neighborhood vehicles that are few hundred meters away. The task of mapping surrounding vehicles mapping can be done by exchanging data in real time between vehicles over dedicated short range communication (DSRC) channels [3]. In addition to communication between vehicles, roadside infrastructure devices can be used to assist vehicles in learning about their environment. For example, they can be used to convey traffic signal or relative information at crosswalks and intersections. The possibility of exchanging real-time information between vehicles at latencies of lower than few hundred milliseconds is a main requirement of a cooperative safety system [3][5][6].

Furthermore, in situational awareness safety applications, information about possible hazards is offered to a vehicle at locations that may be ahead of the vehicle by $0.5-1$ minute. For instance, visibility of the end of a traffic queue is blocked by a road curve, transmitted data between 
vehicles can be used to warn drivers at the distance of a kilometer, alerted drivers should reduce their speed to avoid running into the end of traffic [6].

In fact, cooperative vehicle safety (CVS) systems, also called Cooperative Collision Warning (CCW) systems [4][18], rely on sending and receiving information that contains vehicle state (GPS coordinates, speed, heading, etc.) [2]. CVS Systems communicate with surrounding objects (pedestrians, vehicles and infrastructure) in order to actively evaluate driving situations, and if necessary, generate warnings in different format to support the driver. Information like location, velocity, acceleration, and other vehicle kinematics are expected to be acquiesced by equipment installed in vehicles. This information is conveyed between vehicles through DSRC channel (Figure 1). All vehicles within a transmission range of the sender will be sharing this information. Literatures conclude that in order for CVS to work efficiently, vehicles have to send ten safety messages every second (i.e. $10 \mathrm{~Hz}$ frequency) [7].

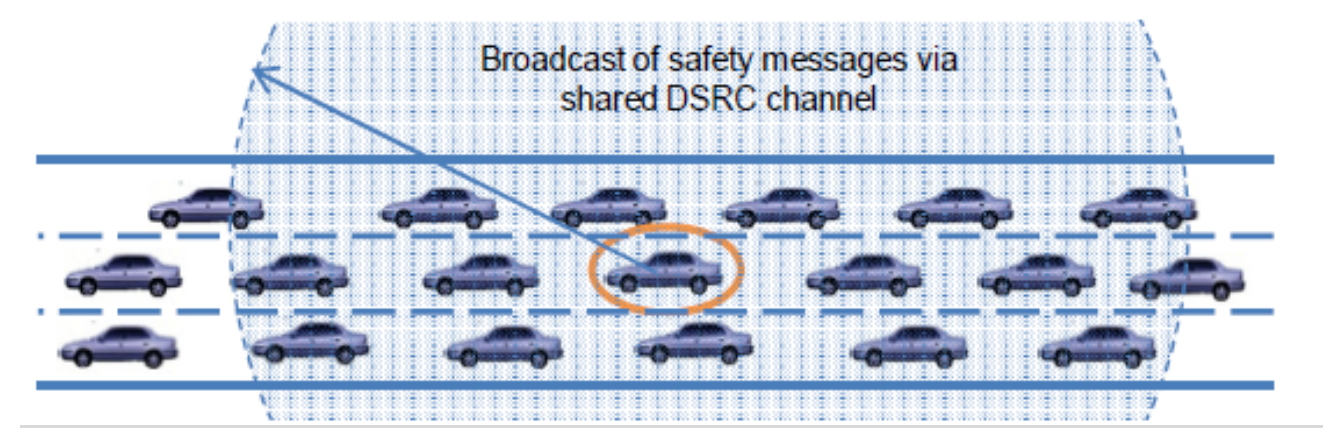

Figure 1. To enable CVS system, each vehicle broadcast its own state info via DSRC

Analysis and improvement of CVS systems involves study of their different components, including, networking, warning generator (hazard detection), and mobility model. These components are traditionally studied separately. In fact, there are no simulation tools that can be used to analyze them together as an integrated framework [2]. As a result, existing tools cannot be used to directly model the mutual effects of these components. On the other hand, correct results 
from simulations to support CVS systems can only be obtained from the appropriate realistic models and components [8].

In this work we considered movement scenarios of two vehicles (a leader and a follower), as they move in a single lane road under different communication conditions. For this CVS system application, we propose augmenting new model in order to achieve better implementation of components interactions by closing the loop of the CVS system framework, and to derive more accurate understanding of CVS systems and to estimate their performance. The added component, named driver reaction's model (DReaM), models the driver's reaction to a hazardous situation.

The rest of the paper is organized as follows. Related work is presented in Section 2. Section 3 highlights major challenges and the open problems, followed by a description of the proposed approach and its component in Section 4. Section 5 presents some initial results. Finally, conclusion and future work are presented in Section 6. 


\section{Related Works}

CVS systems evolved from Active Safety Systems [9]. An Active Safety System is an invehicle system that provides warnings or other forms of assistance to drivers based on in-vehicle sensors such as radar or lidar that can provide some information about the movement of vehicles within their field of operation. It has been argued that CVS system may reduce over $75 \%$ of the nation's crashes [9]. CVS systems aim at accomplishing this goal by using technology alternatives, such as GPS and Dedicated Short Range Communications (DSRC), thus offering the potential to provide information from vehicles that may be occluded from direct line of sight [7].

Studying cooperation between vehicles through data communication started in the late 1980s in the European DRIVE and PROMETHEUS programs, under the label of "cooperative driving" [11]. Researchers from General Motors Europe, in "cooperative foresighted driving" at the 1994 Annual Meeting of ITS America [12], outlined that combined vehicle to vehicle communication and vehicle-roadside transmission to carry information that helps in traffic control, cooperative adaptive cruise control and hazards detection.

Asher and Galler [13][14] in the mid-1990s, developed an idea of cooperative collision warning based on exchanging data between adjacent vehicles, and ranging sensor data. The proposed approach is able to work with a mixture of equipped and unequipped vehicles, since it did not depend only on the exchanged data.

Oloufa and Radwan in [16] proposed using a central server to predict conflict and generate collision avoidance warnings based on DGPS positioning. Sending to and receiving from the server bring about deficiencies such as complexities, latencies and vulnerabilities, which is a weak point to their approach [16]. A similar approach named networked traveler warning system was developed at UC Berkley [17] [18] . In this system, vehicles communicate with a central server 
over $3 \mathrm{G}$ cellular networks to acquire road information. The central server gathers information from a sensor network containing of approximately 600 roadside sensors. This server fuses that with data from other traffic feeds (such as traffic.com) to produce traffic information and warning messages for vehicles.

Bosch in [15] developed a concept for wireless communication of safety information among vehicles within a 1-2 km range, Furthermore, their approach addressed hazardous conditions identified by a variety of means (such as detecting sudden braking activity) rather than relying strictly on differences between the DGPS positioning information as the indicator of a hazard [6][15].

Kato et al. in [19], tested an application of DGPS localization and wireless communication among vehicles to entirely automated driving of a platoon of vehicles on a testing pathway. A similar research approach conducted by Muller et al. [20], where they proposed to use direct communication between vehicles to provide additional information about the surrounding environment such as traffic situations, not only detailed necessary information about vehicle location or speed.

Sengupta et al. [5] introduced a design and prototype of cooperative collision warning systems CCW. Their system relies on wireless communication to enable driver decision support on sub-second timescales. For example, if a vehicle brakes hard, the vehicle behind generates a forward collision warning for its driver within 200 to $500 \mathrm{msec}$. Unlike the sensor based the aim of their $\mathrm{CCW}$ prototype is to enable the same accuracy and time delay response while relying exclusively on rapid wireless broadcast (every $50 \mathrm{~m} \mathrm{sec}$ ) of position by every vehicle [5]. 
Before it is deployed in a real world the vehicular safety application should be thoroughly tested using realistic simulation tools. There are different simulator tools that might be used to perform this task. However, these simulators have different aspects that could be improved. One of the important factors in simulations its realism and considering critical factors that affect the overall results. There are two different approaches researchers have taken to improve the applicability of simulated cooperative safety systems. Some researchers try to improve the existing simulators and their components, for example studies [21] and [22], while others study the effect of cooperating and coupling different components from different vendors, for example studies [23][27] . Researchers have mainly focused on conducting different types of simulations including mobility (traffic), network, and vehicular ad-hoc network (VANET) simulators. Some of the related simulations that had been conducted by the research community are presented in next subsections.

\subsection{Related Work on Mobility Model and Mobility Generator}

There are two main challenges confronted by the study of VANETs: firstly, specifying the exact characteristic of a vehicular mobility model that is able to provide an accurate mobility description at macroscopic and microscopic levels. Secondly, altering this vehicular mobility as a function of the vehicular wireless network. The research community has been trying to tackle these two problems by developing different mobility models [28].

In order for CVS system simulators to provide accurate results, it needs to be provided with a realistic movement model with a sufficient detail fed into vehicular network simulator. This issue is being addressed by many simulators, related work on mobility generator like: SUMO, MOVE, STRAW, FreeSim, and CityMob are briefly discussed in this subsection. 
Michael Behrisch and others [29] presented an overview of a microscopic and continuous traffic simulation package SUMO - Simulation of Urban Mobility. SUMO has been used to study several research topics e.g. route choice and traffic light algorithm or simulating vehicular communication. Therefore, SUMO framework is used to simulate automatic driving or traffic management strategies. It is an open source traffic simulator, which included many useful supporting tools that can be used for different jobs like finding routes, add visualization, networking, and calculating the emission for individual vehicles. For example SUMO was used to give an estimation of the traffic during the Soccer World Cup in 2006. It has many outstanding features including the ability to plug/add custom models to it [29] [30]. SUMO allows working within intermodal; for example, pedestrian's model can be included into a simulation run. It contains many tools used for scenario creation. These tools have features including collision free movement, vehicles with different specification, individual routing, multilane streets, and lane changing [31].

Other researchers like F. Karnadi et al. used SUMO to build their own version of a realistic mobility model simulator named MOVE (MObility model generator for Vehicular networks) [32]. Using MOVE, researchers will be able to generate realistic mobility models for vehicular ad-hoc network simulations. The final stage of running MOVE is to output a mobility trace file with data of realistic vehicle motion. The trace file can be further processed by popular network simulation tools such as ns-3. It has a graphical user interface that makes for quick generation of simulation scenarios, which relieve users from the hassle of programing scripts in addition to learning about the core detail of MOVE [59].

Similar to MOVE, STRAW (STreet RAndom Waypoint) depends on operation of real traffic data [33][34], it uses real US urban maps to originate an accurate simulation results by 
utilizing a vehicular mobility model. STRAW's current implementation is written in java for the highly efficient JiST/SWANS (Scalable Wireless Ad Hoc Network Simulator) discrete-event simulator. The drawback of STRAW is that other network simulators like ns-3 cannot use Straw's mobility trace. STRAW was built in favor of the C3 (Car-to-car cooperation) project, the main idea is to have services conditioned by a cooperative model influenced by the effect of only vehicles that take a part in a particular situation. [34] [35]

Other simulators like FreeSim [36] allows real data to be used within the simulation. It can use either real data, such as that collected by an authority, or lab-produced data to be used to modify weights of edges in map during a simulation phase. In other words, flexibility of using continues and discrete data input qualifies for more accurate and reliable studies of scenarios being analyzed. FreeSim is a macroscopic and microscopic traffic simulator. The simulator provides extensibility by having the ability to use different scripts containing specific instructions to be used during run time.

From the foregoing explanations, it could be noted that FreeSim has the ability to read data. on the other hand, there is CityMob, a simulator which has no ability to deal with stream read data, but it perform different function to CVS system especially providing different mobility models.

In [37] the authors presented a CityMob simulator where they could simulate urban scenarios, including the possibility to model car accidents (represented via non-moving nodes). CityMob has been programed to be compatible with ns-2, it has the capability to generate three different mobility models: Simple Model (SM), Manhattan Model (MM), and realistic Downtown Model (DM). It simulates roads with multiple lanes in each direction, also, cars can be placed in a queue due to traffic congestions. The movement of vehicles is guided and constrained by each road lanes, vehicles are moving at random speed controlled by predefined value. CityMob 
simulates semaphores where they are positioned randomly with different delays. Furthermore, during the simulation traffic is not normally distributed, vehicle within areas with higher density are supposed to move slower than others. Even using the three mobility models (mentioned above) CityMob is not able to consider the inter-vehicle commination [21].

Improving mobility models is not the only goal to implement fully automated CVS system, considering communication is a crucial as well. Wireless networks are the backbone in cooperative safety system, enormous research has been done on this. Next subsection presents related work in network simulators that could be used to simulate vehicular wireless communication networks.

\subsection{Related Work on Network Simulators}

Several network simulators are available today and can be used to model the communication between vehicles in cooperative safety systems. This work presents the main attributes of some of the widely used tools to simulate different scenarios: Ns-3, GloMoSim, and NSN will be discussed respectively.

NS-3 is an event driven network simulator mainly used in research and academic community to test and simulate network protocols and develop network technology. It is a free and available open-source, licensed under the GNU license; it is not an extension of ns-2 simulator rather a new one [38]. Ns-3 manage the simulation engine for researchers to perform their experiments, and it contains many models, of how wired and wireless network components work and perform. It contains an abundance of internal modules and libraries that can be combined with other external libraries. Ns-3 output files can be integrated with other data analysis and visualization tools to give better representation [38].

The idea of modularity is also being used in GloMoSim (Global Mobile system Simulator) [58] where it has been structured in a layered way similar to the seven-layer OSI model. It has the 
ability of parallel discrete-event simulation. Different layers in GloMoSim simulation communicate with each other with well-defined APIs. By using these features, different researchers can integrate their algorithms and models that designed for different layers. It appears in a commercial version called QualNet [23].

While NS-3 and GloMoSim take the advantage of being discrete-event simulators that have well-defined APIs to allow integrating new models, the SNS (a Staged Network Simulator) allows researchers to simulate larger networks in less time.

To improve over both the speed and the scale SNS [42] is proposed to eliminate some redundant computations done by other simulations. Unnecessary operations are done in within a single simulation run and across various runs of the simulator. The staged simulation technique [43] proposes using the caching and reuse technique to reduce unnecessary computations by keeping the output of costly operations to be reused later. In an experiment using a popular wireless network containing fifteen hundreds nodes, due to a staging technique SNS achieves $30 \%$ of the improvement which is a 50 times faster than ns-2. Larger networks can be simulated with this level of performance.

\subsection{Related Work on VANET Simulator}

VANET relies on and is related to two other simulations for its smooth functioning, namely mobility simulation and network simulation. Mobility simulators are mainly used to generate the movement pattern between vehicles. Some of the widely used mobility simulators are discussed in previous subsection. Likewise, Network simulators are used for communication between mobile nodes. [44]

These simulations work independently but to satisfy the need of VANET, a solution is required to use these simulators together [24]. Mobility simulators and Network Simulators are 
planned to be controlled separately without direct interaction. The worlds of Mobility Models and Network Simulators may be compared to "a mute talking to a deaf." [28]. In a VANET If mobility models have the ability to improve network capacity and using the massaging between vehicles to alert the mobility, many promising applications can be achieved in VANET field, these two conditions together are the main reason that holds many improvements of vehicular networks [2] [28].

Different researchers try to address the collaboration and the timing of events in both simulators. For instance, Harri et al. [28] descripted different approaches used to manage the interaction between traffic simulator and network simulator into three cases: Isolated, Embedded, and Federated. In Isolated models case before running the simulation various models should be created and parsed into determined trace format. The main drawback of this approach is difficulty to edit the mobility scenario on later stages during the simulation. In the Embedded models case to make it possible to both simulators to interact with each other one way is to build both of them within a discrete even simulators. The effect of the approach is to get the models to interact natively and efficiently. As a result, the reduced quality of the network simulator is considered as the major drawback of this approach. Federated mobility case way is to use interfaces to allow interaction between network simulators and mobility models. Predefined message format is required to finalize the interaction. This approach allows dynamic interacting and alerting between simulations while they are running in parallel.

Promising VANET simulator is called TraNS (Traffic and Network Simulation Environment) [41], and also aims at federating a traffic simulator SUMO and a network simulator ns-2. TraNS proposed by M. Piorkowski et al. [41] Realistic VANET simulations can be built using the operation modes that TraNS provided. One of its operation modes can address the 
evaluation of the VANET applications that can affect the mobility during the simulation and in real-time. Where it gives the network simulator the ability to control the mobility model, based on the simulated scenario. The coupling between the road traffic and networking simulators is done by using a specific interface, called TraCI [48]; the authors claim it as a generic solution since it has the ability to combine almost all network simulations with traffic simulation [47].

An additional simulator was created to evaluate VANET performance named GrooveSim [45]. It can route the communication within the simulated vehicles, between real vehicles and simulated ones. It was built in a modular architecture way and has the ability to combine variety of models (i.e. mobility and message broadcast models) where they could work on different of link and physical layer communication models. This makes it easy to add other modified models needed for security, applications, networking and interaction between vehicles. The dependencies of the added models are resolved automatically which prevent any conflict with the existing models.

Several researchers preferred to modify an existing simulator to serve their proposed algorithms and techniques. For instance, Joerer et al. [22] used a modified road traffic simulator that allowed selected vehicles to disregard traffic rules, they investigated the impact of safety messaging between cars approaching an intersection. Showing that in suburban environments simple beaconing is not as effective as anticipated. Yet, simple one-hop relaying, e.g., by vehicles parked close to an intersection, can improve drivers' safety substantially [22].

\section{Challenges and Open Problems}

This section lists major challenges and different directions of study that leads to a noteworthy contribution to CVS systems and its related fields. 
- Analyze the effect of communication network on hazardous situation detection (cooperative collision warning $\mathrm{CCW}$ system) in terms of performance.

- Analyze the effect of mobility model(s) on both hazardous situation detection system named forward collision warning (FCW) and performance of communication components (transmission logic, transmission power and rate, packet error rate PER, and network concession control).

- Improve the applicability of simulations by investigating the effect of DReaM on other components in CVS systems.

- Study the effect of integrating different models as a unified approach.

- The effect of mistakenly identifying a hazardous situation (false positive detection) on the behavior of CVS system, or the behavior of platoon of vehicles. In other words, studying the effect of false alerts on a series of vehicles.

- Study the such scenario in which a vehicle (HV) that catches false alert will try to decelerate dramatically and send more data more often which will affect the network and mobility of other vehicles as well.

- Study the change in the driver's preference parameters upon different driving scenarios. For instance, speed limits and desired velocity can be changed within different traffic conditions and/or different weather situations (snow, rainy ... etc.).

In addition to these challenges and open problems, the framework used in this work and its components, which are discussed in the next section, are objectives for more investigation and improvement(s) in order to enhance the accuracy of CVS simulation, and promote their interaction with other existing and potential future models. 


\section{Description of Used Components}

In order to address the challenges described in the previous section and to achieve the research goal, different components are being integrated in this work. This section describes the main components used by the CVS system framework as shown in Figure 2.

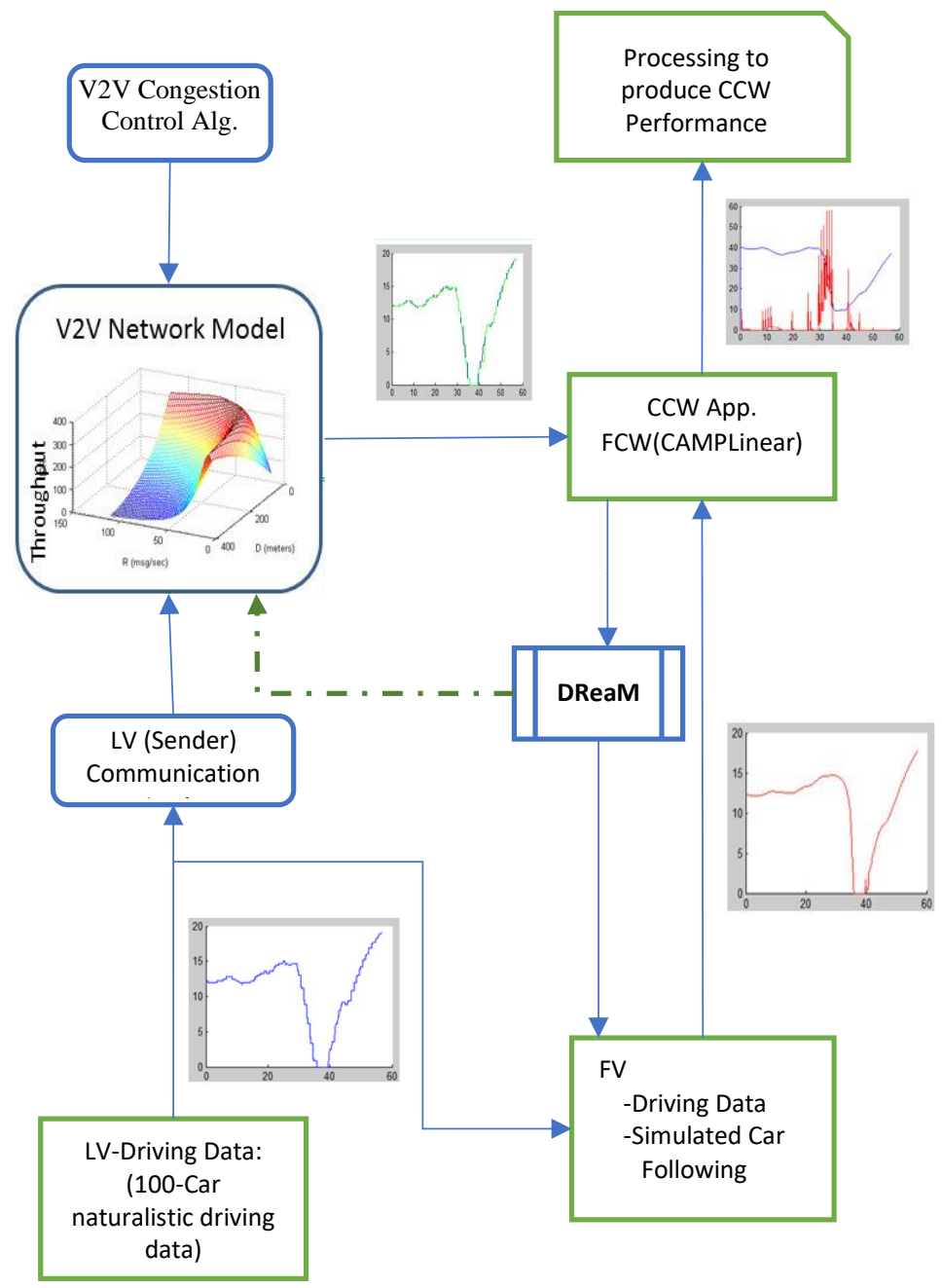

Figure 2 CVS System Framework

CVS system composed of car following model, network model, and warning generation model. These components are described as follows. 


\subsection{Car Following Models}

Car Following Model is one of the widely used models that presents details at motion level. It describes the process of cars following each other in the same lane [27]. In this work, a Car Following Model has been used to represent and control the movement of the vehicles (nodes) during simulation. Following, a brief description of two proven car following models MIT SIMulation program (MITSIM), and Intelligent Driver Model (IDM). In this work both have been used to derive the dynamics of the lagged vehicle (HV), assuming that leading vehicle (RV) dynamics are extracted from the 100-car dataset in [51].

\section{i- MITSIM Car Following Model}

This model is developed by [46], and it is a generalized version of Gazi-Herman-Rothery (GHR) model, where GHR model was developed in the late 50s. The change in acceleration of following vehicle (SV) depends on headway, and the movement of SV that is categorized into three regimes [60];

1) Free flow regimes recognized when the headway of the SV is larger than a predefined threshold $h_{\text {upper }}$ and it does not interact with the preceding vehicle and it permissible to achieve the desired speed. In this regime the acceleration rate is calculating using (1).

$$
a_{n}= \begin{cases}a_{n}^{+} & v_{n}<v_{n}^{\text {desired }} \\ 0 & v_{n}=v_{n}^{\text {desired }} \\ a_{n}^{-} & v_{n}>v_{n}^{\text {desired }}\end{cases}
$$

where $a_{n}^{+}$is the maximum acceleration rate and $a_{n}^{-}$is the normal deceleration rate.

2) Second regime is the emergency regime, occurs if the headway is smaller than a threshold $\mathrm{h}_{\text {lower, }}$, the SV will utilize a deceleration function (2) in order to extend its headway until it becomes greater than $\mathrm{h}_{\text {lower. }}$ 


$$
a_{n}= \begin{cases}\min \left\{a_{n}^{-}, a_{n}-0.5\left(v_{n}-v_{n-1}\right)^{2} /\left(x_{n-1}-L-x_{n}\right)\right\} & v_{n}>v_{n-1} \\ \min \left\{a_{n}^{-}, a_{n-1}+0.25 a_{n}^{-}\right\} & v_{n} \leq v_{n-1}\end{cases}
$$

3) Vehicle is in car-following regime if the headway is between $h_{\text {lower }}$ and $h_{\text {upper }}$ thresholds.

In this case the acceleration rate is calculated using Herman's general car-following model

$$
a_{n}=a^{ \pm} \frac{v_{n}^{\delta^{ \pm}}}{g_{n}^{\gamma^{ \pm}}}\left(v_{n-1}-v_{n}\right)
$$

where $\mathrm{a}_{n}$ is the acceleration, $v_{n}$ is the speed of the $\mathrm{SV}, \mathrm{g}_{\mathrm{n}}$ the space gap between vehicles, and $v_{n-1}$ is the speed of the leading vehicle. $\alpha, \beta$, and $\gamma$ are model parameters. $(+)$ and (-) are used for accelerating situations and decelerating cases, respectively.

\section{ii- Intelligent Driver Model (IDM)}

This model was developed by [56], and it calculates the acceleration $v^{\prime}$ as a continuous function of velocity $v$, gap $s$ and velocity difference $\Delta v$ as:

$$
\dot{v}_{f}\left(s, v_{f}, \Delta v\right)=a\left[1-\left(\frac{v_{f}}{v_{0}}\right)^{\delta}-\left(\frac{s^{*}\left(v_{f}, \Delta v\right)}{s}\right)^{2}\right]
$$

where the subscript $f$ denotes the following vehicle (i.e. SV), and the subscript $\mathrm{p}$ denotes the leading vehicle $(\mathrm{RV}), \Delta v(t)$ velocity deference at time $(\mathrm{t}), S$ is the separation distance between vehicles, and $\mathrm{S}^{*}$ is the desired minimum gap which formulated as:

$$
s^{*}\left(v_{f}, \Delta v\right)=s_{0}+s_{1} \sqrt{\frac{v_{f}}{v_{0}}}+T v_{f}+\frac{v_{f} \Delta v}{2 \sqrt{a b}}
$$

where $T$ is a constant that denotes desired safety time gap, $a$ and $b$ are the maximum acceleration and desired deceleration respectively.

\subsection{Network Model}

Vehicular network communication could be modeled at different levels of complexity and congestion control algorithms. A stochastic model of the cooperative vehicular network in [56] 
can be used in this research to determine the probability of receiving a message at different distances from a sender for a range of parameters, such as rate (frequency) and range (power) of transmission. A packet error rate $P E R=E(R, P, \rho, d)$ can be used to evaluate the packet loss rate in a road with density $\rho$, for rate $R$ and power $P$, and at distance $d$ from the sender [49].

Congestion control algorithms is not the main concern of this work. Alternatively, this work uses modeling the vehicular network with a random loss rate (PER) and inflict it on the messages transferred between vehicles.

\subsection{Communication Logic (Policies)}

The component that defines the way that DSRC network is using to interchange messages between nodes called communication logic. It controls both the time in which a message can be transmitted and the power associated with each transmission. Choice of higher power value will allow message to cover wider area. The choice of a higher power rate results in a higher chance of interference, consequently, PER value will be higher at all nodes.

This work uses two prominent logics used by community: Periodic Beaconing (PB) [3], and Error-Dependent policy (ED) ( [7] inspired by [49]). The periodic beaconing (PB) policy uses a simple technique of periodically sampling the signal and transmitting it over the channel. In error-dependent transmission, a sender locally simulates the estimator of the receiver (remote estimator) to understand receiver's error in estimating sender's position. The sender sends a new message to correct the receiver's estimator every time the estimating error exceeds a threshold. Significant improvement can be achieved by this logic. For instance, reducing the required messages needed to preserve the estimation error value at the recipient [7]. 


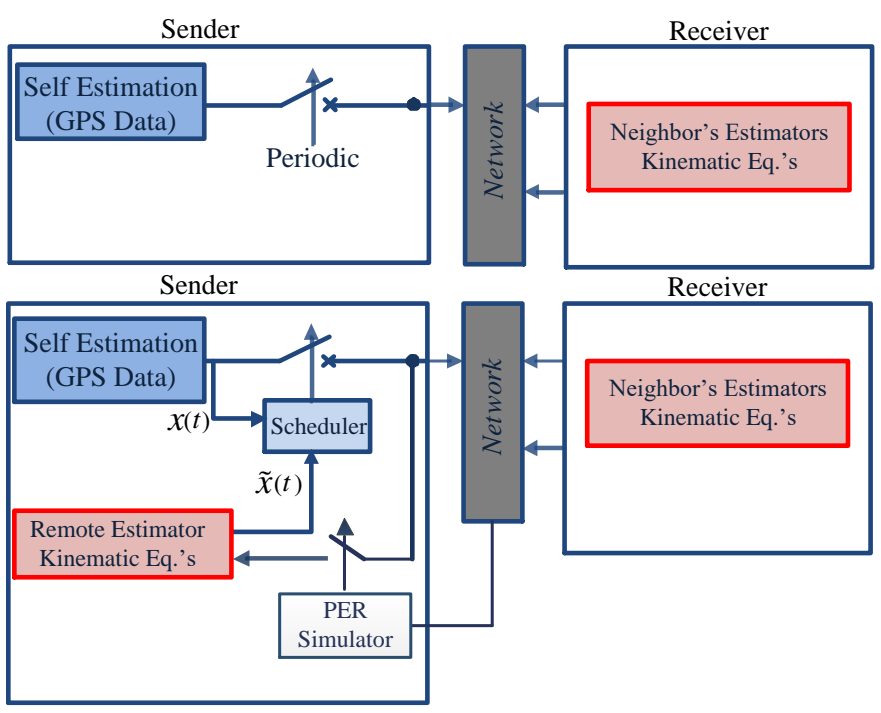

Figure 3. Communication logic: (top) Periodic Beaconing, (bottom) Network- Aware Error-Dependent.

\subsection{Safety Algorithm}

For this study we used the CAMPLinear algorithm in [50] to generate warnings to the following vehicle. The algorithm designed in such way that an alert is issued as soon as it becomes necessary for an action to be taken by the driver of the following vehicle. The alert timing continuously changes as vehicles delivered data changes. The alert timing described as a "warning range". A collision warning is issued to the FV whenever the separation distance between vehicles becomes less than the warning range $(r w)$. The warning range, $\mathrm{r}_{\mathrm{w}}$, derivative is outlined here for a brief discussion, further details in [50]. $r w$ can be seen as a function of speed and acceleration of both leading and following vehicles (i.e., $L V_{v}, L V_{a}, F V_{V}$, and $F_{V a}$ ), $r w$ is calculated as a sum of Brake Onset Range (BOR) and driver system reaction range $r d$ :

$$
\begin{aligned}
& r w=B O R n+r d, \\
& r_{d}=\left(v_{F V}-v_{L V}\right) t_{d}+0.5\left(a_{F V}-a_{L V}\right) t_{d}^{2},
\end{aligned}
$$

where $t_{d}$ is the driver and brake system reaction delay $(\sim 2.5 \mathrm{~s})$. BOR is computed for three different scenarios

1) Leading vehicle stationary at the beginning and end of scenario 


$$
B O R_{1}=\left(v_{F V P}\right)^{2} /\left(-2 d_{r q d}\right),
$$

2) Leading vehicle moving at the beginning and end of scenario

$$
B O R_{2}=\left(v_{F V P}-v_{L V P}\right)^{2} /\left(-2\left(d_{r q d}-d_{L V}\right)\right),
$$

3) Leading vehicle moving at the beginning but stopping at the end

$$
B O R_{3}=\frac{v_{F V P}^{2}}{-2 d_{r q d}}-\frac{v_{L V P}^{2}}{-2 d_{L V}},
$$

where $v_{F V P}$ and $v_{L V P}$ are the estimated velocity and calculated as:

$$
v_{F V P}=v_{F V}+a_{F V} t_{d} \text {, and } v_{L V P}=v_{L V}+a_{L V} t_{d},
$$

and $d_{L V}$ is the deceleration of $\mathrm{LV} ; d_{r q d}$ is the required deceleration by $\mathrm{FV}$ for avoiding a crash and is modeled as follows (in $f t / s^{2}$ ):

$$
\mathrm{d}_{\mathrm{rqd}}=-5.3+0.68 \mathrm{a}_{\mathrm{LV}}+2.57\left(\mathrm{v}_{\mathrm{LV}}>0\right)-0.086\left(\mathrm{v}_{\mathrm{FV}}-\mathrm{v}_{\mathrm{LVP}}\right)
$$

The assumption here is that FV has an accurate information about its state (speed, position, and acceleration), and an estimated information about LV. Figure 5 shows the results for a sample scenario from the 100-car dataset that is in [51]. Here, the above equations have been applied to derive the warning range. If the distance between vehicles becomes less than the warning range, the driver is expected to receive an alert issued by the warning generation algorithm. 


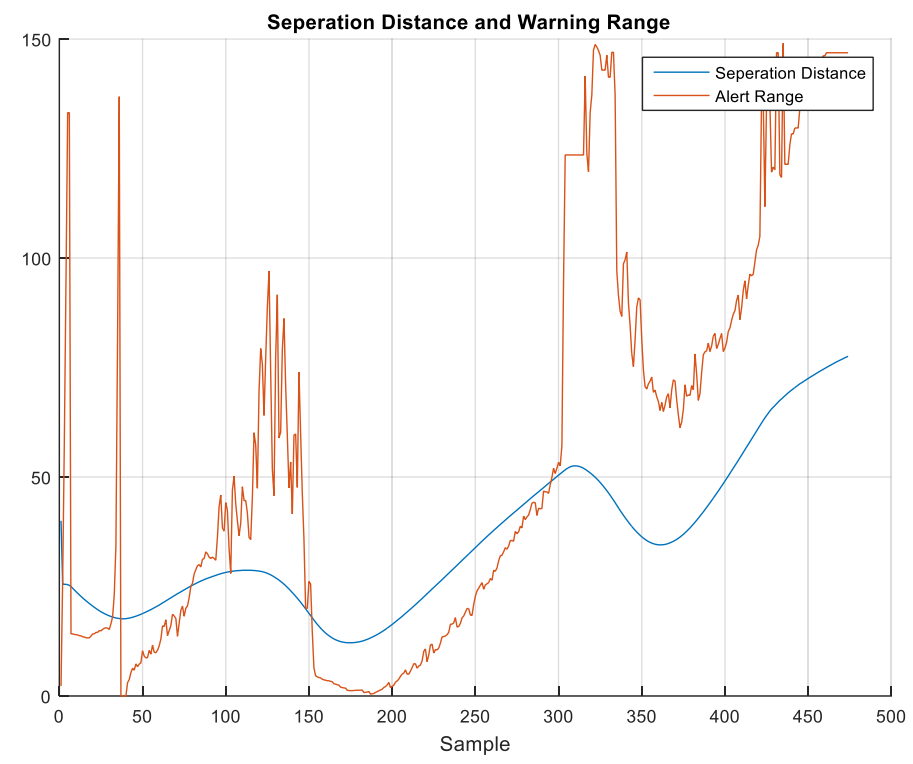

Figure 4 Warning Range derived by CAMPLinear algorithm

\subsection{Safety Algorithm Performance Metrics}

Warning algorithm to be continuously detecting situational threat state every $T_{c w}$ seconds ( $T_{c w}$ in this study is $100 \mathrm{~ms}$ ). To study the effect of different system parameters a metric that can indicate how well the system is performing is needed. Even though there is no standard metric that can be used to measure the performance of FCW algorithms, this work uses a method presented in [52] for quantifying the performance of FCW. The method in [52] uses confusion matrix to evaluate measurement values as false positive, false negative, precision, true positive $(T P)$, true negative, and Accuracy (A). Since these evaluation metrics provide similar evaluation results two of them are used for evaluation in this paper, True Positive and Accuracy are defined as:

$$
\begin{aligned}
& \mathrm{TP}=\mathrm{C}_{\mathrm{T}} /\left(\mathrm{I}_{\mathrm{S}}+\mathrm{C}_{\mathrm{T}}\right), \\
& \mathrm{A}=\left(\mathrm{C}_{\mathrm{T}}+\mathrm{C}_{\mathrm{S}}\right) /\left(\mathrm{I}_{\mathrm{S}}+\mathrm{I}_{\mathrm{T}}+\mathrm{C}_{\mathrm{T}}+\mathrm{C}_{\mathrm{s}}\right),
\end{aligned}
$$

where $C_{\mathrm{T}}$ is the number of correctly predicted threads, $C_{\mathrm{s}}$ is the number of correctly predicted safe indications, $I_{\mathrm{s}}$ is the number of mistakenly predicted safe indications, and $I_{\mathrm{T}}$ is the number of 
mistakenly predicted hazard indications. Accuracy gives an overall measure of the accuracy of the system in generating or not generating a warning [52].

\subsection{Dataset Used}

Results showed later in this report obtained with the input data from the 100-car naturalistic driving field test [51]. The dataset includes hundreds vehicle dynamics of crash or near to crash. The dynamics of the FV is derived using a proven car-following model MITSIM developed by [46], described above, assuming that LV dynamics are extracted from the 100-car dataset.

\subsection{Driver Reaction Model (DReaM)}

DReaM emulates the reaction taken by vehicle's driver once he/she gets an alert. The normal reaction taken at the last minute by the driver to avoid crash with front vehicle is hard brake. The proposed reaction model induce/stimulate hard braking in order to avoid crashing with other vehicle(s). It is a straightforward model to evaluate the framework presented in Figure 2.

Deceleration value set by this model is equivalent to the required deceleration value suggested by McLaughlin at el. In [54] and used in the Knipling algorithm, the deceleration values is $0.6 \mathrm{~g}\left(\right.$ or $\left.5.88 \mathrm{~m} / \mathrm{s}^{2}\right)$.

\section{Initial Results}

Initial results and graphs obtained using the framework and the models discussed earlier can be divided into five subsections.

\subsection{Effect of Communication on Delivered Information}

Figure 5 shows the effect that communication network (DSRC) has on delivered information (position, speed, and acceleration), information about leading vehicle is either received through the network model or estimated using the estimation method. By using the 
constant acceleration estimation method figure 5 shows that the used system has better estimation of leader's acceleration than its velocity
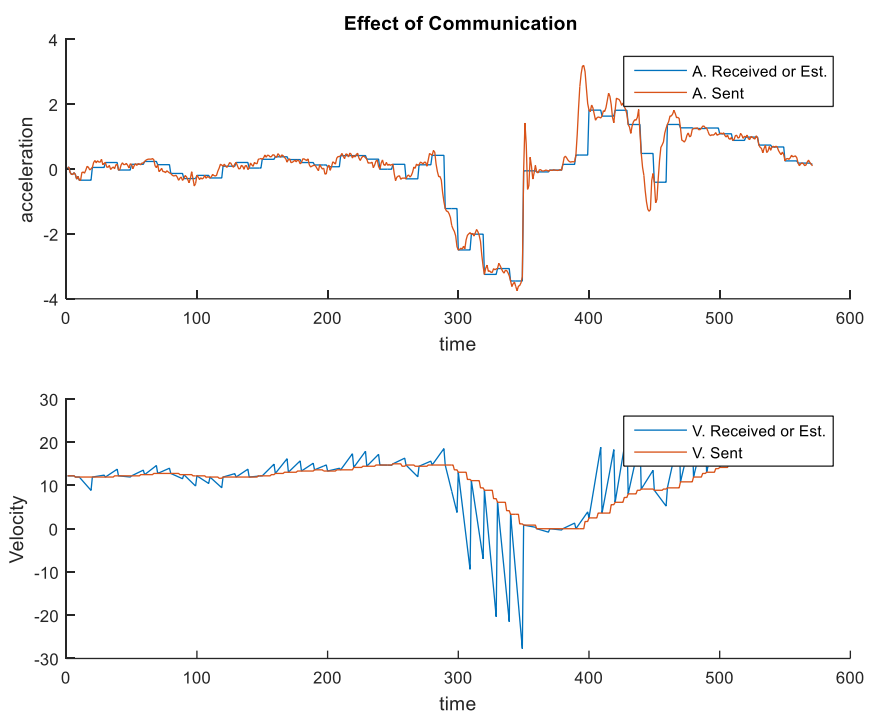

Figure 5 the effect on received acceleration and velocity data

\subsection{Effect of Communication on Position Estimation of Leading Vehicle}

Figures 6 shows the effect of losing messages through communication channel on the position tracking error PTE. This figure is plotted using averaging of position tracing errors data from all dataset scenarios versus the packet error rate (PER) and rate of delivered information, respectively. As a natural result of error in estimation of close vehicles both car following model and reaction model will behave in a senseless way, which causes more accidents; therefore the results of simulated scenario will be different than the real data field. 

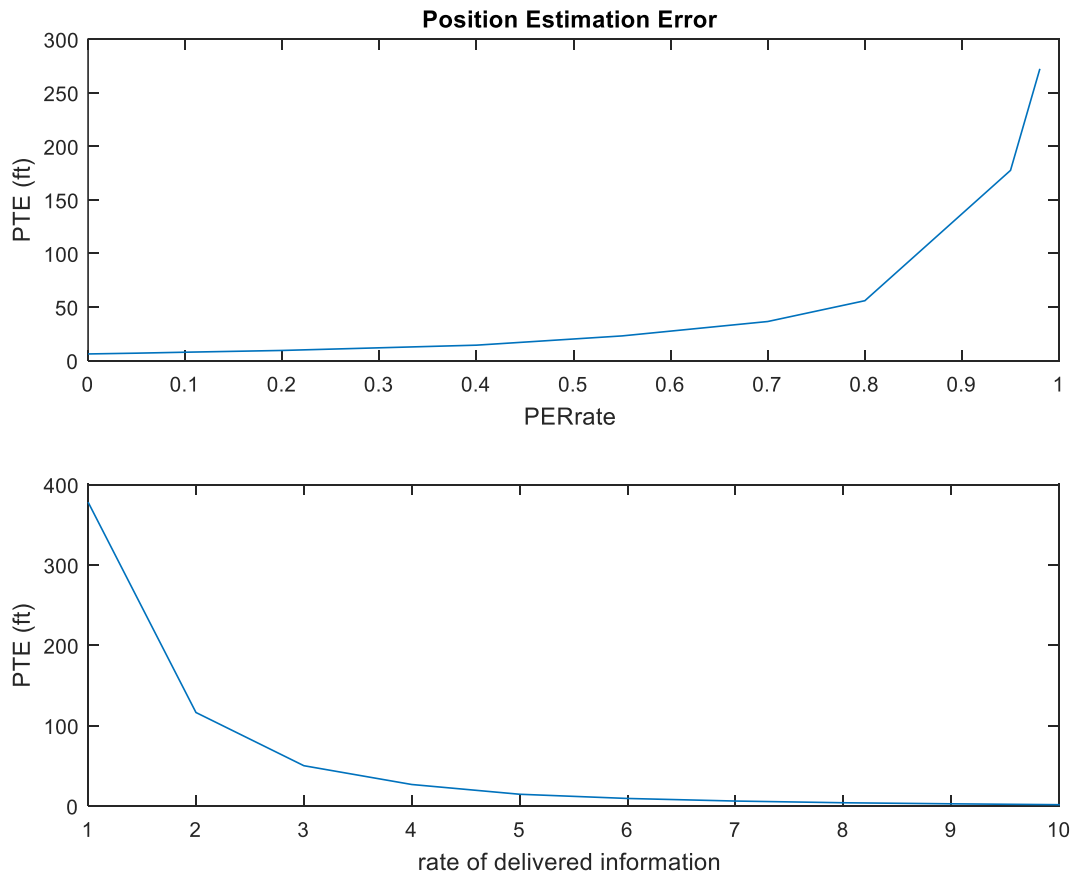

Figure 6 Effect of networking on Estimation of Leading vehicle's Position

\subsection{Effect of Communication on Performance of Hazard Detection Algorithm}

The effect of communication on the performance of warning algorithm (CAMPLinear) is illustrated in figures 7,8 , and 9 . The method presented in[52] is used to evaluate values of warning accuracy, true positive (TP), false positive FP, and false negative FN. These values are plotted in the said figures, and they are calculated from all dataset scenarios versus both delivered information and packet error rate (PER), respectively. 

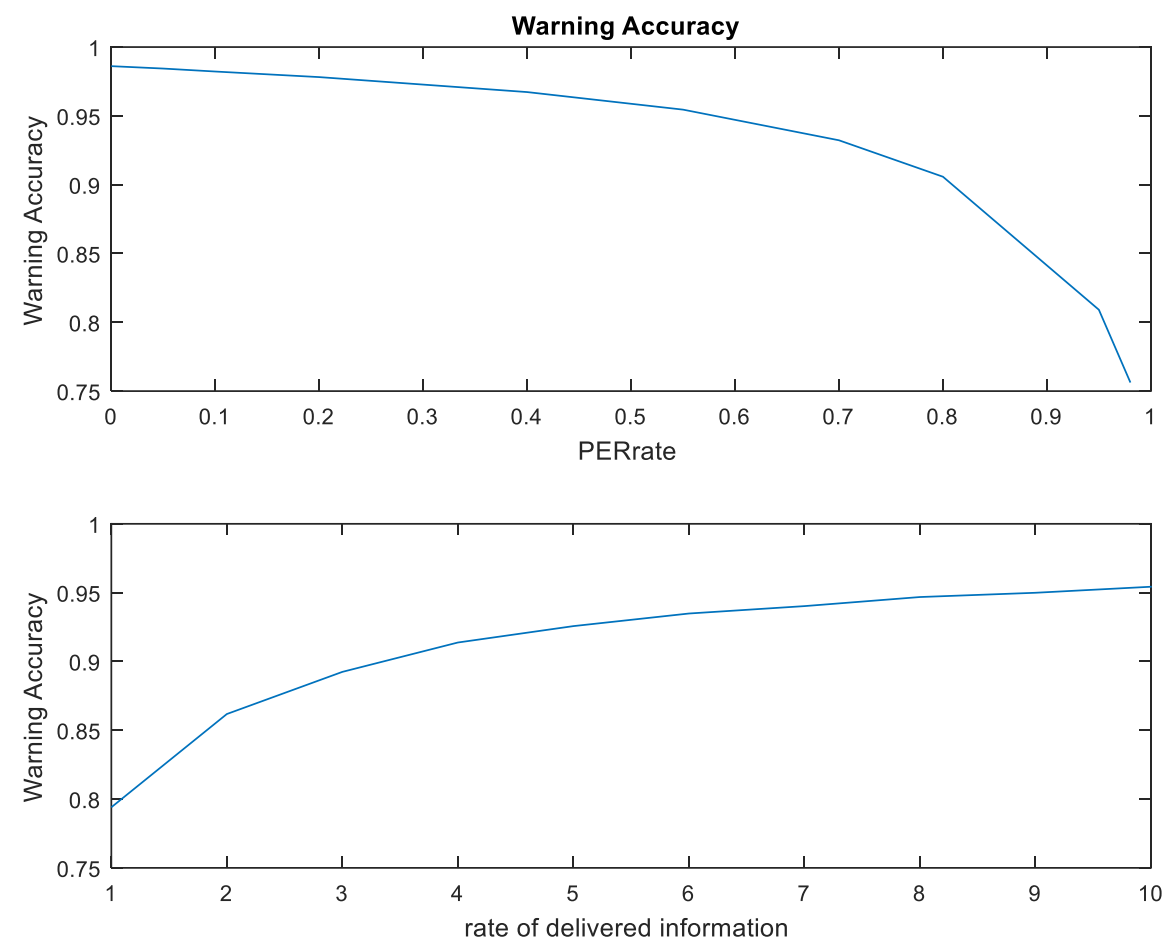

Figure 7 FCW (CAMPLinear) Accuracy

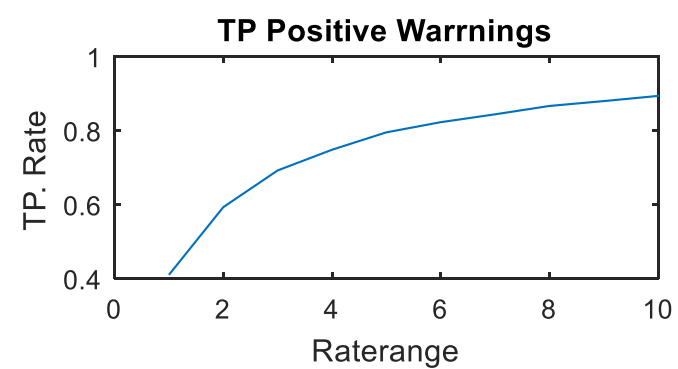

6. False Positive Warrnings

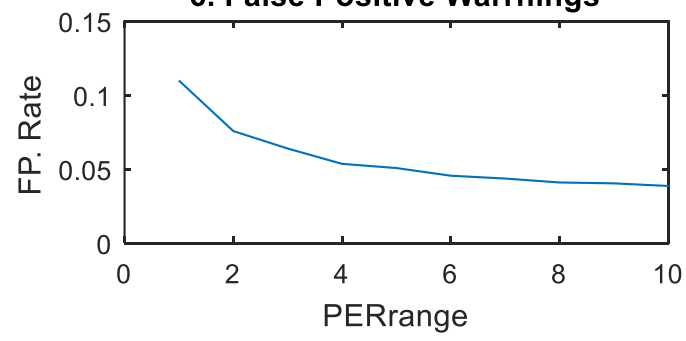

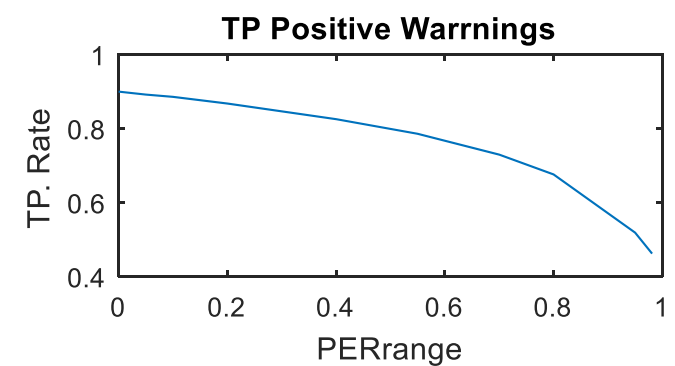

5. False Positive Warrnings

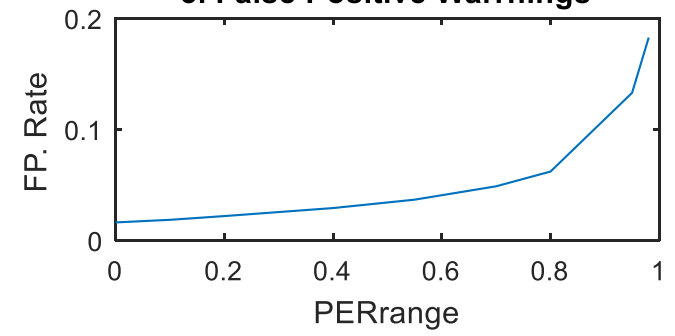

Figure 8 Performance of FCW (CAMPLinear) 

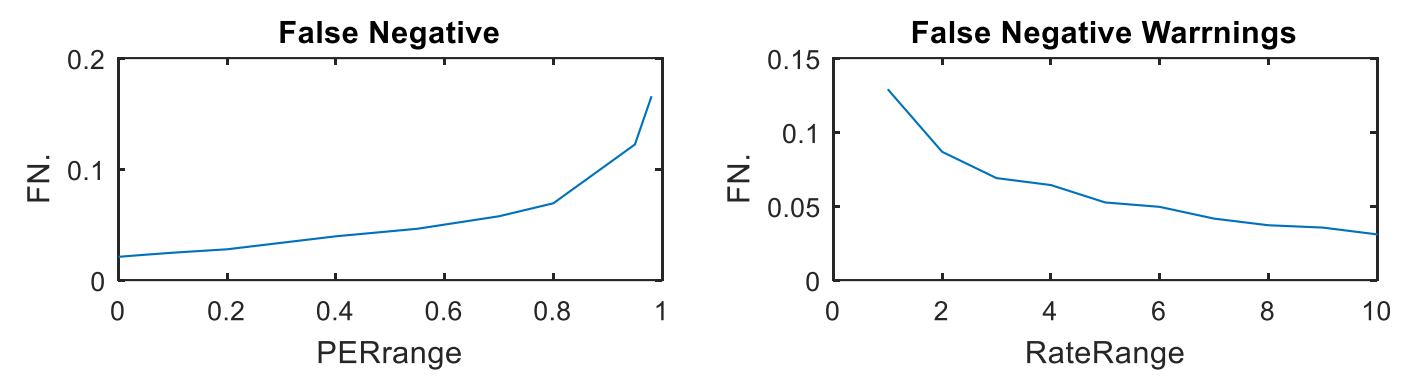

Figure 9 Performance of FCW (CAMPLinear)

\subsection{Effect of Networking and DReaM Combination on vehicles Movement and Positioning of Vehicles}

Figure 12 illustrates the effect of adding DReaM to CVS system. Augmenting the DReaM will affect both movement and positioning of vehicles, and it will result in increasing the separation distance between vehicles. Curves in this figure are plotted with the assumption of no losses through the communication channel between vehicles and a high delivery rate of information. The following (lag) vehicle tries to maintain the desired speed while keeping a safe distance that is enough to decelerate if a FCW alert is issued. 


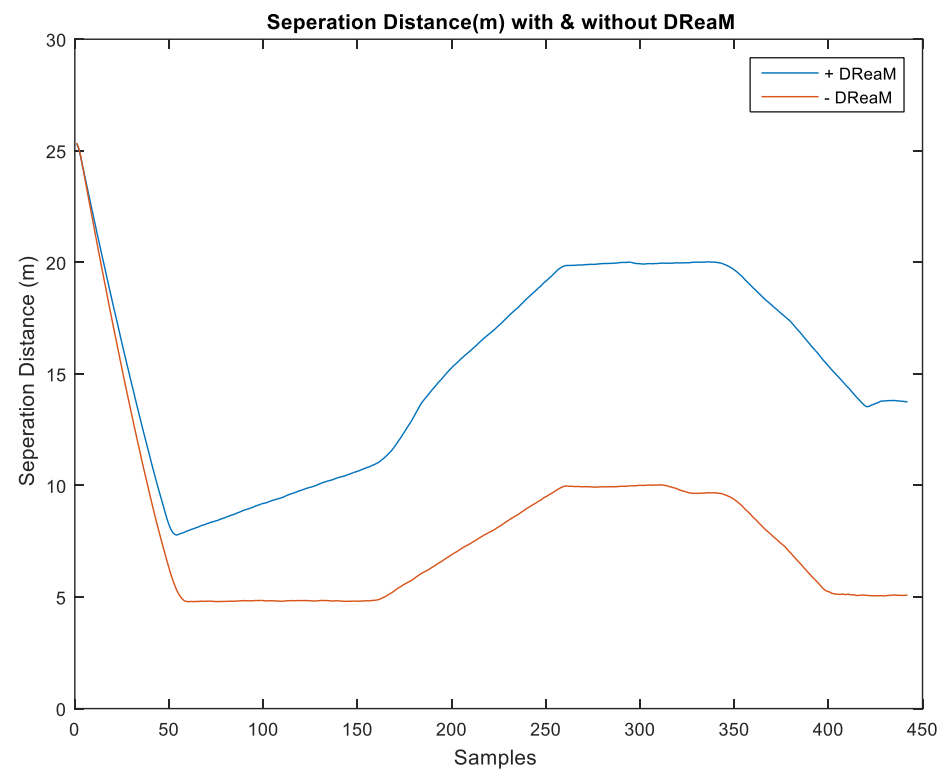

Figure 10 Separation distance between vehicles

\subsection{Effect of DReaM}

Figures 13, 14 and 15 summarize the effect of DReaM. Both boxplots in Figure 12 represent the total number of hazardous situations detected by cooperative collusion warning algorithm (i.e. CAMPLinear). At every hazardous situation an alert is provided to both CFM and DReaM regardless if there is a true hazard (true positive) or if it is a mistake by the warning algorithm (false positive). In either way a reaction to that alert has to be taken. Both boxplots shows that the number of alerts will increase as the ratio of missing information increases. This relation is true regardless of the reason that causes lose of information. In other words, the network throughput has a direct impact to the number of issued alerts. 

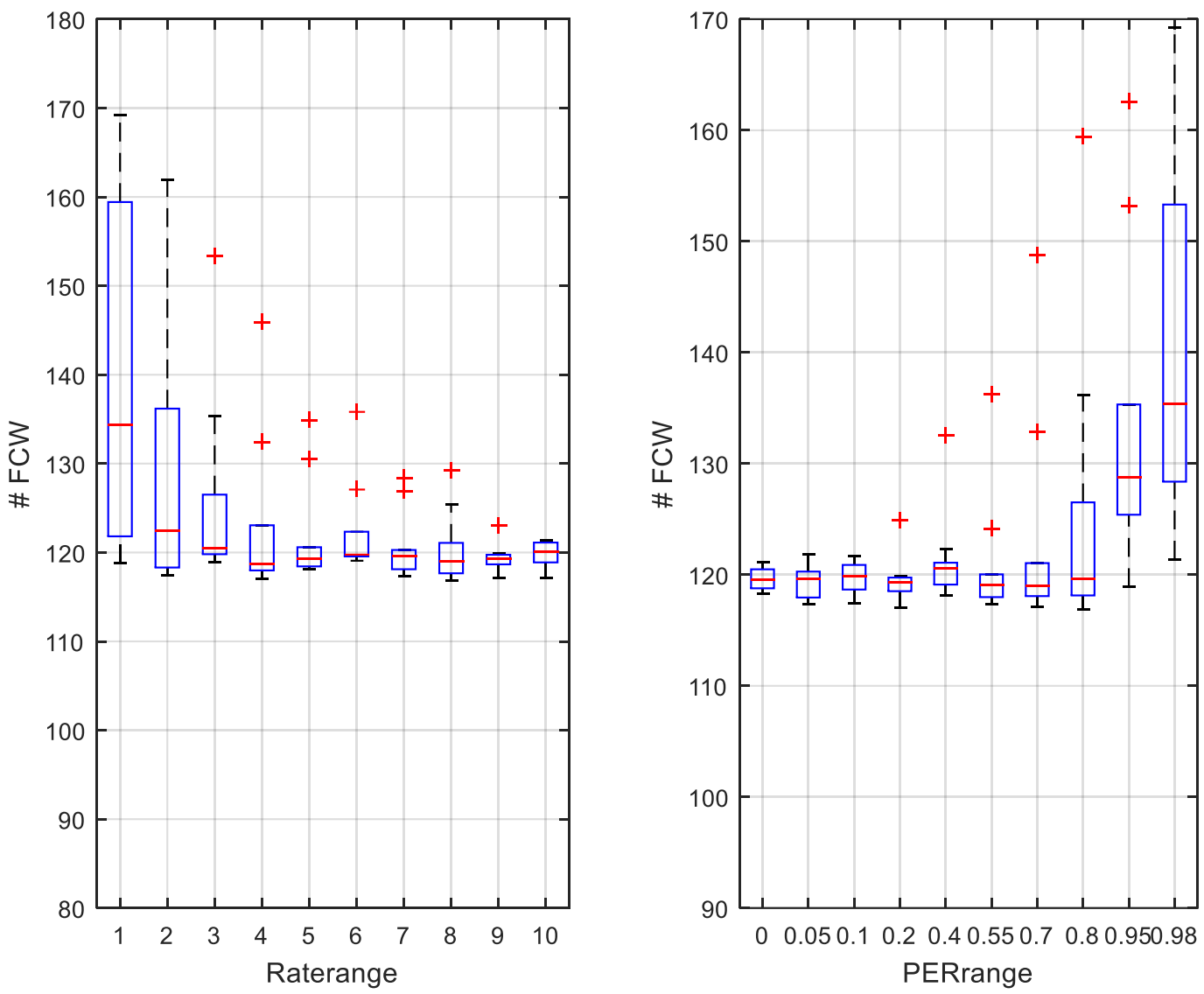

Figure 11 number of generated alerts by CAMPLinear

Figure 13 illustrates the relation between the number of reactions taken by DReaM and the rate of delivered information and packet error rate. For better illustration both boxplots in (a) and (b) are redrawn as a ratio to their total number of response in (c) and (d), respectively. 


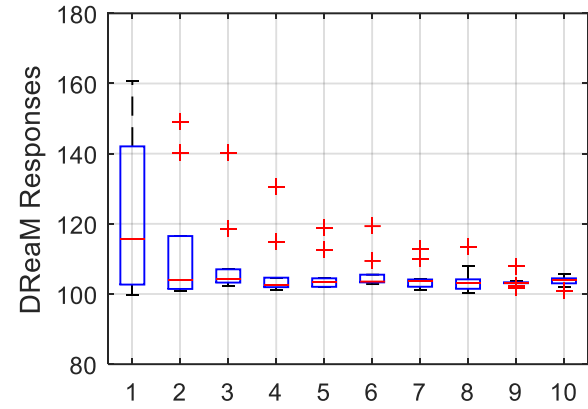

(a) Raterange

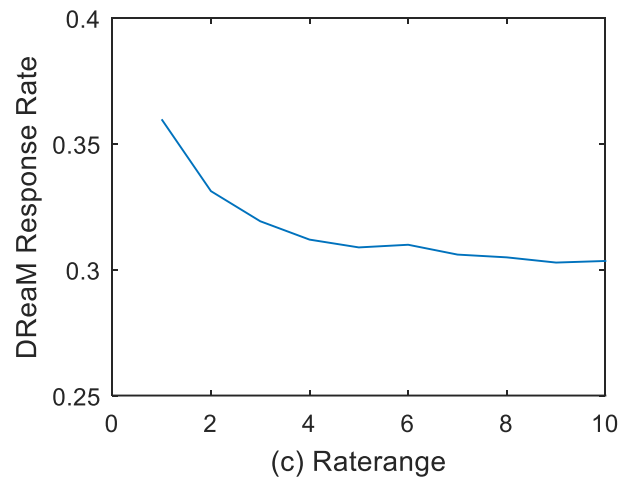

(c) Raterange

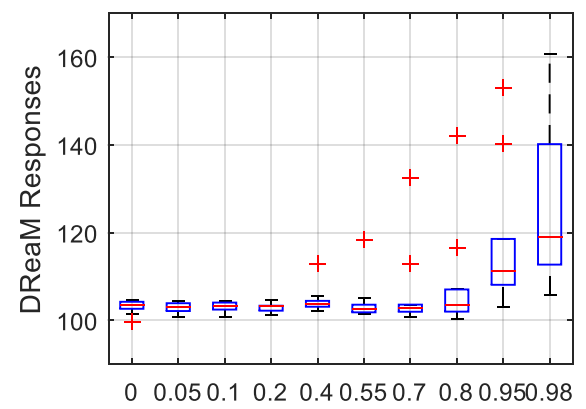

(b) PERrange

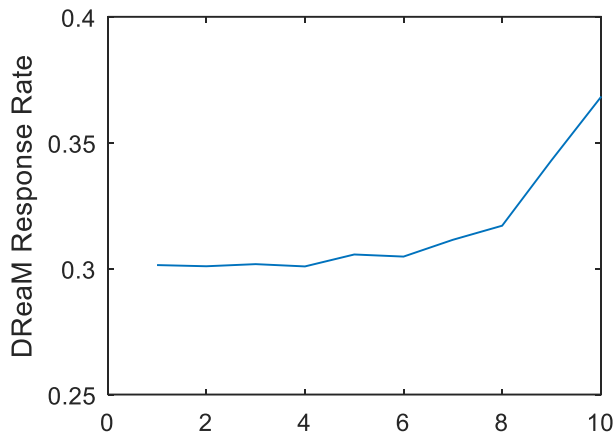

(d) Raterange

Figure 12 Reactions Taken by DReaM

DReaM did not provide reactions to all alerts shown in Figure 13. Instead, the reaction (deceleration) taken by car following model (MITSIM) is sufficient to keep the following vehicle in a safe situation and maintain a safe time to crash. A summary of these actions is presented in Figure 15. This figure shows the number of situations where there is an alert and CFM (MITSIM) acted well, and its deceleration value is suitable without the need of implementing DReaM. 

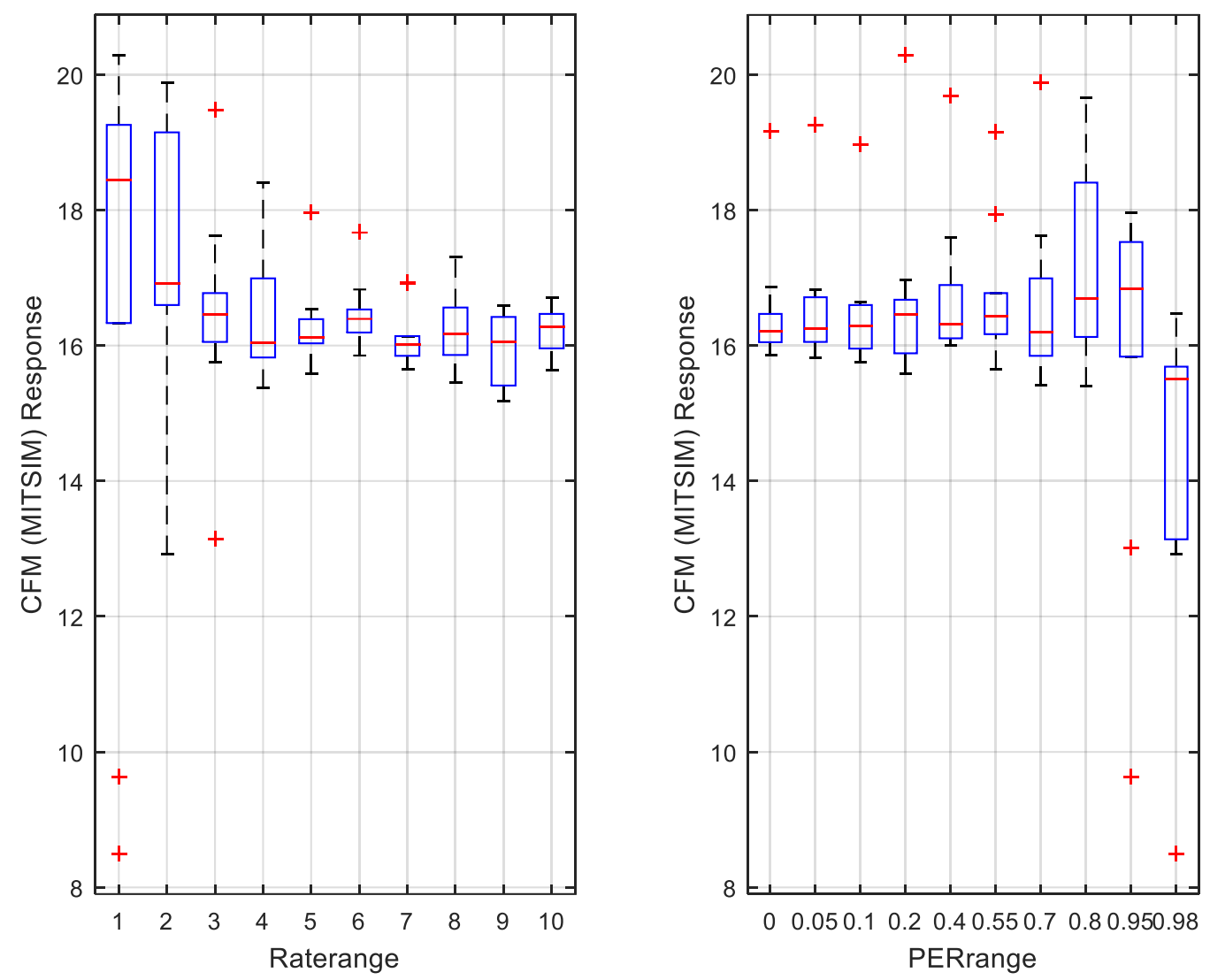

Figure 13 Actions by CFM (MITSIM)

\section{Conclusions}

We examined a simulation modeling framework that describes cooperative vehicle safety system as one unified model. The presented framework is powered by cooperation and communication between vehicles. Investigated components are communication model, mobility model, warning generation, and driver response to warning indicating an emergency of near to crash situation.

A large dataset of naturalistic driving scenarios, along with mentioned components, were used to investigate the effects of different components of this system. In this work widely used 
mobility model (MITSIM) is used to derive the dynamics of the following vehicle (HV), assuming that leading vehicle (RV) dynamics are extracted from the dataset.

This work studied the synthesis components of CVS system and the effect of its main components on each other. It aims to analyze adding another model to simulate the reaction of human receiving an alert from a warning component. The obtained results prove that DReaM is an important component that can affect the simulation results and should be considered in building realistic simulations and implementing CVS applications.

The proposed framework paves the way to build a simulation that composed of realistic components for CVSS-type applications in VANETs as well. For instance, the presented model can be used to investigate the effect of reaction to alerts (i.e. DReaM) on the changes of separation gap(s) between platoon vehicles.

The work presented here can be expended in different aspects and it has a room for more improvements. Our prospective future work for this research involves study and analysis of the following.

- The mutual effect of communication logic and driver reactions on the rate of hard braking.

- The effect of DReaM on movement and separation distance(s) between vehicles in a platoon.

- Different applied deceleration by the DReaM could be calculated as a function of time to crash (TTC).

- Study the effect of Adding more models. For instance, Driver Distraction Model (DDM) like one proposed in [55] can be added to the framework to examine the effect of distraction on other main system components. 


\section{References}

[1] Yaser P. Fallah "Impact of V2V Communication Protocols and Performance on Reliability of Cooperative Collision Warning Systems," ACM ICCPS2014, April 14-18, 2014, Berlin, Germany.

[2] Moradi-Pari, E., Gani, S., Fallah, Y., Naserian, M. et al., "Co-Simulation of Cooperative Vehicle Safety Applications and Communication Networks," SAE Int. J. Passeng. Cars Electron. Electr. Syst. 8(2):344-349, 2015, doi: 10.4271/2015-01-0285.

[3] Wireless Access in Vehicular Environment (WAVE) in Standard 802.11, Specific Requirements: IEEE 802.11p/D1.0, Feb. 2006.

[4] Vehicle Safety Communications Consortium (VSCC), "Vehicle Safety Communications Project, Task 3 Final Report: Identify Intelligent Vehicle Safety Applications Enabled by DSRC," 2005.

[5] R. Sengupta, S. Rezaei, S. E. Shladover, J. A. Misener, S. Dickey, H.Krishnan, "Cooperative Collision Warning Systems: Concept Definition and Experimental Implementation," Journal of Intelligent Transportation Systems, vol.11,No.3, pp. 143-155, 2007.

[6] Y. P. Fallah, C.L. Huang, R. Sengupta and H. Krishnan, "Design of Cooperative Vehicle Safety Systems based on Tight Coupling of Communication, Computing and Physical Vehicle Dynamics", in Proc. of ACM/IEEE Int. Conf. on Cyber-Physical Systems, ICCPS 2010.

[7] Rezaei, Shahram, et al. "Tracking the Position of Neighboring Vehicles using Wireless Communications." Transportation Research Part C 18.3 (2010): 335-350.

[8] He, Ying, et al. "Accident Driver Model for Vehicular Ad-Hoc Network Simulation." Vehicle Power and Propulsion Conference (VPPC), 2013 IEEE. IEEE, 2013.

[9] NHTSA, ITS Joint Program Office, 1997. Report to congress on the national highway traffic safety administration ITS program, program progress during 1992-1996 and strategic plan for 1997-2002. US Department of Transportation, Washington, DC, January 1997.

[10] Godbole, D.N., Sengupta, R., Misener, J., et al, 1998. Benefit evaluation of crash avoidance systems. Transportation Research Record No: 1621 (January), 1-9.

[11] F. Broqua, G. Lerner, V. Mauro, and E. Morello, "Cooperative Driving: Basic Concepts and a First Assessment of 'Intelligent Cruise Control' Strategies", Proceedings of Advanced Telematics in Road Transport, DRIVE Conference, Vol. II, pp. 908-929, February 1991.

[12] E. Schubert, L. Reck and J. Graf, "Communication Systems for Cooperative Foresighted Driving”, Proceedings of Fourth Annual Meeting of ITS America, pp. 374 - 381, Atlanta, GA, April 1994.

[13] H. J. Asher and B.A. Galler, "Collision Warning Using Neighboring Vehicle Information", Proceedings of Sixth Annual Meeting of ITS America, pp. 674 - 684., Houston, TX, April 1996.

[14] H. J. Asher, B.A. Galler, "Collision Warning in a Mix of Equipped and Unequipped Vehicles", Proceedings of Fourth World Congress on Intelligent Transport Systems, Berlin, November 1997.

[15] C. Passmann, C. Brenzel and R. Meschenmoser, "Wireless Vehicle to Vehicle Warning System", SAE Paper No 2000-01-1307, in Intelligent Vehicle Systems, SAE Special Publication SP-1538, pp. 149- 160. 2000.

[16] Oloufa, A.E. Radwan, "Server-Based Collision Avoidance Using DGPS", Proceedings of Eighth World Congress on Intelligent Transport Systems, Sydney, October 2001. 
[17] C. L. Huang et al., "Information Dissemination Control for Cooperative Active Safety Applications in Vehicular Ad-Hoc Networks," Proc. IEEE Globecom 2009, Nov. 2009.

[18] R. Sengupta et al., "Cooperative Collision Warning Systems: Concept Definition and Experimental Implementation,” J. Intelligent Transportation Systems, vol. 11, no. 3, July 2007, pp. 143-55.

[19] S. Kato, S. Tsugawa, K. Tokuda, T. Matsui, and H. Fujii, "Vehicle Control Algorithms for Cooperative Driving with Automated Vehicles and Intervehicle Communications", IEEE Transactions on Intelligent Transportation Systems, Vol. 3, No. 3, pp. 155-161, September 2002.

[20] S. Muller, M. Uchanski, and J.K. Hedrick, "Cooperative Estimation Using Vehicle Communication”. Vehicle System Dynamics, Vol. 39, No. 2, pp. 121-133, February 2003.

[21] He, Ying, et al. "Accident Driver Model for Vehicular Ad-Hoc Network Simulation." Vehicle Power and Propulsion Conference (VPPC), 2013 IEEE. IEEE, 2013.

[22] S. Joerer, M. Segata, B. Bloessl, R. L. Cigno, C. Sommer and F. Dressler, "To crash or not to crash: Estimating its likelihood and potentials of beacon-based IVC systems," Vehicular Networking Conference (VNC), 2012 IEEE, Seoul, 2012, pp. 25-32.

[23] Martinez, Francisco J., et al. "A Survey and Comparative Study of Simulators for Vehicular Ad Hoc Networks (VANETs)." Wireless Communications and Mobile Computing 11.7 (2011): 813-28.

[24] Khairnar, Vaishali D., and Pradhan, S. N. "Comparative Study of Simulation for Vehicular Ad-hoc Network." International Journal of Computer Applications (0975 - 8887) Volume 4No.10, August 2010

[25] Thiemann, C., M. Treiber, and A. Kesting. "Estimating Acceleration and Lane-Changing Dynamics from Next Generation Simulation Trajectory Data." TRANSPORTATION RESEARCH RECORD 2088.2088 (2008): 90-101.

[26] F. Dressler, C. Sommer, D. Eckhoff and O. K. Tonguz, "Toward Realistic Simulation of Intervehicle Communication," in IEEE Vehicular Technology Magazine, vol. 6, no. 3, pp. 4351, Sept. 2011.

[27] Hassan, A., 2009. VANET Simulation. Högskolan i Halmstad. Master Thesis and Technical Report.

[28] Harri, J., F. Filali, and C. Bonnet. "Mobility Models for Vehicular Ad Hoc Networks: A Survey and Taxonomy." IEEE Communications Surveys \& Tutorials 11.4 (2009): 19-41.

[29] M. Behrisch, L. Bieker, J. Erdmann, D. Krajzewicz, Sumo - simulation of urban mobility: an overview, in: SIMUL 2011, The Third International Conference on Advances in System Simulation, Barcelona, Spain, 2011.

[30] http://www.dlr.de/ts/en/desktopdefault.aspx/tabid-9883/16931_read-41000/

[31] Krajzewicz, D., and C. Rossel. "Simulation of Urban MObility (SUMO). German Aerospace Centre, 2007." (2007)

[32] F. K. Karnadi, Z. H. Mo and K. c. Lan, "Rapid Generation of Realistic Mobility Models for VANET," Wireless Communications and Networking Conference, 2007. WCNC 2007. IEEE, Kowloon, 2007, pp. 2506-2511.

[33] David R. Choffnes and Fabián E. Bustamante. Modeling Vehicular Traffic and Mobility for Vehicular Wireless Networks, Tech. Report NWU-CS-05-03, Department of Computer Science, Northwestern University, 2005. 
[34] David R. Choffnes, Fabián E. Bustamante, An integrated mobility and traffic model for vehicular wireless networks, Proceedings of the 2nd ACM international workshop on Vehicular ad hoc networks, September 02-02, 2005, Cologne, Germany

[35] Zheng, Q., Hong, X., and Ray, S. Recent advances in mobility modeling for mobile ad hoc network research. In ACM-SE 42: Proceedings of the 42nd annual Southeast regional conference (New York, NY, USA, 2004), ACM Press, pp. 70-75.

[36] J. Miller and E. Horowitz, "FreeSim - a free real-time freeway traffic simulator," Intelligent Transportation Systems Conference, 2007. ITSC 2007. IEEE, Seattle, WA, 2007, pp. 18-23.

[37] F. J. Martinez, J. C. Cano, C. T. Calafate and P. Manzoni, "CityMob: A Mobility Model Pattern Generator for VANETs," Communications Workshops, 2008. ICC Workshops '08. IEEE International Conference on, Beijing, 2008, pp. 370-374

[38] A. Wang and W. Jiang, "Research of Teaching on Network Course Based on NS-3," Education Technology and Computer Science, 2009. ETCS '09. First International Workshop on, Wuhan, Hubei, 2009.

[39] https://www.nsnam.org/docs/release/3.24/tutorial/singlehtml/index.html

[40] http://jist.ece.cornell.edu

[41] JiST: Embedding Simulation Time into a Virtual Machine. Proceedings of EuroSim Congress on Modelling and Simulation, September 2004

[42] http://www.cs.cornell.edu/people/egs/sns/

[43] Kevin Walsh , Emin Gün Sirer, Staged simulation: A general technique for improving simulation scale and performance, ACM Transactions on Modeling and Computer Simulation (TOMACS), v.14 n.2, p.170-195, April 2004

[44] Mitul K. Patel, "Comparative Study of Vehicular Ad-hoc Network Mobility Models and Simulators", Volume 47- No.6, International Journal of Computer Applications (0975 - 888), June 2012.

[45] Rahul Mangharam, Daniel S. Weller, Daniel D. Stancil, Ragunathan Rajkumar, Jayendra S. Parikh, GrooveSim: a topography-accurate simulator for geographic routing in vehicular networks, Proceedings of the 2nd ACM international workshop on Vehicular ad hoc networks, September 02-02, 2005, Cologne, Germany.

[46] Yang, Q., 1997. A Simulation Laboratory for Evaluation of Dynamic Traffic Management Systems. Department of Civil and Environmental Engineering, Massachusetts Institute of Technology: 193.

[47] M. Piórkowski , M. Raya , A. Lezama Lugo , P. Papadimitratos , M. Grossglauser , J.-P. Hubaux, TraNS: realistic joint traffic and network simulator for VANETs, ACM SIGMOBILE Mobile Computing and Communications Review, v.12 n.1, January 2008

[48] http://sumo.sf.net/wiki/index.php/TraCI

[49] Y.P. Fallah, M. Kalantari-Khandani," Analysis of the Coupling of Communication network and Safety Application in Cooperative Collision Warning Systems", in proc. 2015 ACM /IEEE Int. Conference on Cyber-Physical Systems (ICCPS), pp. 228-237, 2015

[50] Y. Xu, J. Hespanha, "Estimation under uncontrolled and controlled communication in Networked Control Systems," Proc. of Conf.Decision and Control, Dec. 2005.

[51] Kiefer, R., Cassar, M. T., Flannagan, C. A., LeBlanc, D. J., Palmer, M. D., Deering, R. K., \& Shulman, M. A. 2003. "Forward Collision Warning Requirements Project: Refining the CAMP Crash Alert Timing Approach by Examining 'Last-Second' Braking and Lane Change Maneuvers Under Various Kinematic Conditions”. Report No. DOT-HS-809-574. Washington, DC: National Highway Traffic Safety Administration. 
[52] Lee, K., and H. Peng. "Evaluation of automotive forward collision warning and collision avoidance algorithms." Vehicle System Dynamics 43.10 (2005): 735-751.

[53] Dingus, T. A., Klauer, S. G., Neale, V. L., Peterson, A., Lee, S. E., Sudweeks, J., Perez, M. A., Hankey, J., Ramsey, D., Gupta, S., Bucher, C., Doerzaph, Z. R., Jarmeland, J., \& Knipling, R. R. 2006. "The 100-Car naturalistic driving study, Phase II - Results of the 100-Car field experiment." Washington, DC: National Highway Traffic Safety Administration.

[54] S. B. McLaughlin, J. M. Hankey, T. A. Dingus, and S. G. Klauer, "Development of an FCW Algorithm Evaluation Methodology With Evaluation of Three Alert Algorithms, Tech. Rep. June, 2009.

[55] Salvucci, Dario D. "Modeling Driver Behavior in a Cognitive Architecture." Human Factors: The Journal of the Human Factors and Ergonomics Society 48.2 (2006): 362-.

[56] Treiber M, Hennecke K, Helbing D (2000) Congested traffic states in empirical observations and microscopic simulations. Phys Rev E 2(2):1805-1824.

[57] Y. P. Fallah, CL Huang, R. Sengupta, H. Krishnan, "Analysis of Information Dissemination in Vehicular Ad-Hoc Networks with Application to Cooperative Vehicle Safety Systems", IEEE Trans. on Vehicular Technology, Vol 60, Issue 1, pp. 233-247, Jan 2011.

[58] X. Zeng, R. Bagrodia and M. Gerla, "GloMoSim: A Library for Parallel Simulation of LargeScale Wireless Networks", Proceedings of the 1998 Workshop on Parallel and Distributed Simulation, pp. 154-161

[59] https://www3.epa.gov/otaq/models/moves/

[60] Olstam, Johan Janson, and Andreas Tapani. "Comparison of Car-following models." Swedish National Road and Transpost Research Institute.-2004.-45 c. 